\title{
Prognostic effect of different PD-L1 expression patterns in squamous cell carcinoma and adenocarcinoma of the cervix
}

\author{
A Marijne Heeren ${ }^{1,2}$, Simone Punt ${ }^{3}$, Maaike CG Bleeker ${ }^{4}$, Katja N Gaarenstroom ${ }^{5}$, \\ Jacobus van der Velden ${ }^{6}$, Gemma G Kenter ${ }^{1,6,7}$, Tanja D de Gruijl2 ${ }^{2}$ and \\ Ekaterina S Jordanova ${ }^{1,3}$
}

\begin{abstract}
${ }^{1}$ Center for Gynecological Oncology Amsterdam (CGOA), Department of Obstetrics and Gynecology, VU University Medical Center, Amsterdam, The Netherlands; ${ }^{2}$ Department of Medical Oncology, VU University Medical Center-Cancer Center Amsterdam, Amsterdam, The Netherlands; ${ }^{3}$ Department of Pathology, Leiden University Medical Center, Leiden, Leiden, The Netherlands; ${ }^{4}$ Department of Pathology, VU University Medical Center, Amsterdam, The Netherlands; ${ }^{5}$ Department of Gynecology, Leiden University Medical Center, Leiden, Leiden, The Netherlands; ${ }^{6}$ Center Gynecological Oncology Amsterdam (CGOA), Department of Obstetrics and Gynecology, Academic Medical Center, Amsterdam, The Netherlands and ${ }^{7}$ Center Gynecological Oncology Amsterdam (CGOA), Department of Gynecology, Netherlands Cancer Institute-Antoni van Leeuwenhoek, Amsterdam, The Netherlands
\end{abstract}

\begin{abstract}
Programmed death-ligand 1 (PD-L1) is expressed in various immune cells and tumor cells, and is able to bind to PD-1 on T lymphocytes, thereby inhibiting their function. At present, the PD-1/PD-L1 axis is a major immunotherapeutic target for checkpoint inhibition in various cancer types, but information on the clinical significance of PD-L1 expression in cervical cancer is largely lacking. Here, we studied PD-L1 expression in paraffin-embedded samples from two cohorts of patients with cervical cancer: primary tumor samples from cohort I (squamous cell carcinoma, $n=156$ and adenocarcinoma, $n=49$ ) and primary and paired metastatic tumor samples from cohort II (squamous cell carcinoma, $n=96$ and adenocarcinoma, $n=31$ ). Squamous cell carcinomas were more frequently positive for PD-L1 and also contained more PD-L1-positive tumor-associated macrophages as compared with adenocarcinomas (both $P<0.001$ ). PD-L1-positive tumor-associated macrophages were found to express CD163 and/or CD14 by triple fluorescent immunohistochemistry, demonstrating an M2-like phenotype. Interestingly, disease-free survival $(P=0.022)$ and disease-specific survival $(P=0.046)$ were significantly poorer in squamous cell carcinoma patients with diffuse PD-L1 expression as compared with patients with marginal PD-L1 expression (i.e., on the interface between tumor and stroma) in primary tumors. Disease-specific survival was significantly worse in adenocarcinoma patients with PD-L1-positive tumorassociated macrophages compared with adenocarcinoma patients without PD-L1-positive tumor-associated macrophages $(P=0.014)$. No differences in PD-L1 expression between primary tumors and paired metastatic lymph nodes were detected. However, PD-L1-positive immune cells were found in greater abundance around the metastatic tumors as compared with the paired primary tumors $(P=0.001$ for squamous cell carcinoma and $P=0.041$ for adenocarcinoma). These findings point to a key role of PD-L1 in immune escape of cervical cancer, and provide a rationale for therapeutic targeting of the PD-1/PD-L1 pathway.
\end{abstract}

Modern Pathology (2016) 29, 753-763; doi:10.1038/modpathol.2016.64; published online 8 April 2016

Cervical cancer is the fourth most common cancer among women worldwide and is induced by a

Correspondence: Dr ES Jordanova, $\mathrm{PhD}$, Center for Gynaecological Oncology Amsterdam (CGOA), Department of Obstetrics and Gynecology, VU University Medical Center, Free University Amsterdam, De Boeleaan 1117, Amsterdam 1081 HV, The Netherlands.

E-mail: e.jordanova@vumc.nl

Received 10 December 2015; revised 13 February 2016; accepted 27 February 2016; published online 8 April 2016 persistent infection with one of the high-risk strains of the human papilloma virus (HPV), most frequently HPV16 and/or HPV18. ${ }^{1,2}$ Several types of cervical tumor histology can be distinguished, but the most commonly applied stratification is squamous cell carcinoma vs adenocarcinoma, both with different oncogenic mutations, ${ }^{3,4}$ and different immunological tumor microenvironment. ${ }^{5-7}$ Despite these substantial differences, current treatment modalities are the same for both squamous cell carcinomas and 
adenocarcinomas. ${ }^{8}$ At present, patients with cervical cancer are treated with radical hysterectomy and pelvic lymphadenectomy or chemoradiation, depending on tumor stage and tumor size. ${ }^{8-10}$ Unfortunately, the number of patients with adenocarcinoma is still rising and these patients seem to have a poorer survival rate than squamous cell carcinoma patients, especially if adenocarcinoma present with tumor-positive lymph nodes. ${ }^{11-14}$ To improve the prognosis of cervical cancer patients, novel immunotherapeutic strategies need to be developed and established. In addition, histological subtype-specific treatment needs to be considered, which requires a detailed investigation of the tumor microenvironment in relation to clinical outcome of these tumor types.

Promising immunotherapeutic therapies targeting immune checkpoint molecules, such as cytotoxic T-lymphocyte-associated protein 4 (CTLA-4) and programmed cell death protein 1 (PD-1) expressed on activated $\mathrm{T}$ cells, counteract the immunosuppressive cycle prevailing in the tumor microenvironment and have led to complete and long-lasting clinical responses. ${ }^{15,16}$ Also, anti-programmed cell death ligand 1 (PD-L1) therapy has been associated with improved survival outcome in several types of cancer, including lung cancer, melanoma, renal cell cancer, and bladder cancer. ${ }^{17,18}$ At present, in advanced cervical cancer, clinical phase I/II trials are ongoing examining the effects of ipilimumab (anti-CTLA-4; NCT01711515), pembrolizumab (antiPD-1; NCT02054806), and nivolumab (anti-PD-1; NCT02488759); however, no study results have been reported yet.

Recently, we have identified a suppressive myeloid cell subset expressing PD-L1, with high and interrelated rates of regulatory $\mathrm{T}$ cells in metastatic lymph nodes of patients with cervical cancer. ${ }^{19}$ Currently, information is largely lacking about PD-L1 expression patterns in primary and metastatic cervical tumors. Therefore, we investigated the expression of PD-L1 in primary and metastatic cervical cancer in relation to the two major histological subtypes (squamous cell carcinoma and adenocarcinoma), and studied the correlation with pathological and clinical characteristics in two patient cohorts. This study provides more insight into the role of PD-L1 in cervical cancer, and strengthens the rationale for blocking the PD-L1/ PD-1 immunosuppressive axis.

\section{Materials and methods}

\section{Study Group}

Formalin-fixed, paraffin-embedded material was collected from two different patient cohorts. Patient cohort I consisted of 156 squamous cell carcinomas and 49 adenocarcinomas primary tumor samples from the Leiden University Medical Center (Leiden,
Table 1 Clinicopathological characteristics of patient cohort I

\begin{tabular}{|c|c|c|}
\hline Clinicopathological characteristics & $S C C$ & $A C$ \\
\hline Number of patients & 156 & 49 \\
\hline \multicolumn{3}{|l|}{ Age } \\
\hline Mean & 48 & 41 \\
\hline Min & 22 & 26 \\
\hline Max & 87 & 72 \\
\hline \multicolumn{3}{|l|}{ FIGO stage ${ }^{\mathrm{a}}$} \\
\hline IBI & $93(59.5)$ & $41(84)$ \\
\hline$\geq \mathrm{IBII}$ & $62(40)$ & $8(16)$ \\
\hline Missing & $1(0.5)$ & $0(0)$ \\
\hline \multicolumn{3}{|l|}{$H P V$ status $^{\mathrm{a}}$} \\
\hline HPV 16 & $97(62)$ & $18(37)$ \\
\hline HVP18 & $24(15)$ & $19(39)$ \\
\hline Other & $25(16)$ & $4(8)$ \\
\hline Negative & $10(7)$ & $8(16)$ \\
\hline \multicolumn{3}{|l|}{ Tumor size ${ }^{\mathrm{a}}$} \\
\hline$\leq 4 \mathrm{~cm}$ & $61(39)$ & $39(80)$ \\
\hline$>4 \mathrm{~cm}$ & $74(47)$ & $10(20)$ \\
\hline Unknown & $21(14)$ & $0(0)$ \\
\hline \multicolumn{3}{|l|}{ Parametrium invasion $^{\mathrm{a}}$} \\
\hline Yes & $26(17)$ & $4(8)$ \\
\hline No & $128(82)$ & $45(92)$ \\
\hline Unknown & $2(1)$ & $0(0)$ \\
\hline \multicolumn{3}{|l|}{ Lymph node metastases $^{\mathrm{a}}$} \\
\hline Yes & $50(32)$ & $13(27)$ \\
\hline No & $105(67.5)$ & $36(73)$ \\
\hline Unknown & $1(0.5)$ & $0(0)$ \\
\hline \multicolumn{3}{|l|}{ Recurrence within 5 years $^{\mathrm{a}}$} \\
\hline Yes & $45(29)$ & $15(31)$ \\
\hline No & $111(71)$ & $34(69)$ \\
\hline
\end{tabular}

Abbreviations: AC, adenocarcinoma; FIGO, International Federation of Gynecology and Obstetrics; SCC, squamous cell carcinoma.

${ }^{a}$ Data shown as $n(\%)$.

The Netherlands), and patient cohort II consisted of 96 squamous cell carcinomas and 31 adenocarcinomas paired primary and metastatic tumor samples from the Academic Medical Center (Amsterdam, The Netherlands), VU University Medical Center (Amsterdam, The Netherlands), or Leiden University Medical Center (Leiden, The Netherlands). Patients in both cohorts underwent surgery as primary treatment between 1985-2008 and the patient characteristics are shown in Tables 1 and 2, respectively. Patient samples were handled and used in accordance with the medical ethical guidelines described in the Code of Conduct for Proper Secondary Use of Human Tissue of the Dutch Federation of Biomedical Scientific Societies.

\section{Immunohistochemistry}

Immunohistochemical staining was performed with an automated Ventana immunostainer (Ventana Medical Systems, Tucson, AZ, USA) as previously 
Table 2 Clinicopathological characteristics of patient cohort II

\begin{tabular}{|c|c|c|}
\hline Clinicopathological characteristics & $S C C$ & $A C$ \\
\hline Number of patients & 96 & 31 \\
\hline \multicolumn{3}{|l|}{ Age } \\
\hline Mean & 44 & 41 \\
\hline Min & 24 & 23 \\
\hline Max & 81 & 66 \\
\hline \multicolumn{3}{|l|}{ FIGO stage s,b $^{\mathrm{a}, \mathrm{b}}$} \\
\hline IBI & $58(60)$ & $16(52)$ \\
\hline$\geq \mathrm{IBII}$ & $37(39)$ & $15(48)$ \\
\hline Missing & $1(1)$ & $0(0)$ \\
\hline \multicolumn{3}{|l|}{ Tumor size ${ }^{\mathrm{a}}$} \\
\hline$\leq 4 \mathrm{~cm}$ & $27(28)$ & $11(36)$ \\
\hline$>4 \mathrm{~cm}$ & $68(71)$ & $29(61)$ \\
\hline Unknown & $1(1)$ & $1(3)$ \\
\hline \multicolumn{3}{|l|}{ Parametrium invasion $^{\mathrm{a}}$} \\
\hline Yes & $35(37)$ & $8(26)$ \\
\hline No & $59(61)$ & $22(71)$ \\
\hline Unknown & $2(2)$ & $1(3)$ \\
\hline \multicolumn{3}{|l|}{ Lymph node metastases ${ }^{\mathrm{a}}$} \\
\hline Yes & $96(100)$ & $31(100)$ \\
\hline No & $0(0)$ & $0(0)$ \\
\hline \multicolumn{3}{|l|}{ Recurrence within 5 years ${ }^{\mathrm{a}}$} \\
\hline Yes & $30(31)$ & $14(45)$ \\
\hline No & $61(64)$ & $13(42)$ \\
\hline Missing & $5(5)$ & 4 (13) \\
\hline
\end{tabular}

Abbreviations: AC, adenocarcinoma; FIGO, International Federation of Gynecology and Obstetrics; SCC, squamous cell carcinoma.

Data shown as $n(\%)$.

${ }^{\mathrm{b}} \mathrm{NB}$ : HPV status is not known for this patient cohort.

described using Cell Conditioning 1 Solution (Ventana Medical Systems) as antigen retrieval, 1:200 rabbit anti-PD-L1 antibody for $48 \mathrm{~min}$ at $36^{\circ} \mathrm{C}$ (clone E1L3N; Cell Signaling, Danvers, MA, USA), and using the OptiView DAB IHC Detection Kit (Ventana Medical Systems). ${ }^{20}$

For triple immunofluorescence staining on four squamous cell carcinoma patients from cohort I, 1:100 rabbit anti-PD-L1 (clone SP142; Spring Bioscience, Pleasanton, CA, USA), 1:25 mouse IgG2a anti-CD14 (clone 7; Abcam, Cambridge, UK), and 1:100 mouse IgG1 anti-CD163 (clone 10D6; Novocastra, Milton Keynes, UK) were used and detected with Alexa Fluor 647 goat anti-rabbit, Alexa Fluor 546 goat anti-mouse IgG2a, and Alexa Fluor 488 goat anti-mouse IgG1 (all from Life Technologies, Grand Island, NY, USA), as described previously. ${ }^{20}$

\section{Imaging, Scoring, and Analysis}

The immunohistochemically PD-L1-stained slides were analyzed and imaged using a bright-field microscope (Olympus BX50; Olympus, Center Valley, PA, USA). Tumor fields were distinguished from normal tissue by the use of nuclear staining with hematoxylin. Primary and metastatic tumor cells were designated PD-L1 positive, when $\geq 5 \%$ of the tumor cells were positive for PD-L1. Moreover, in both primary and metastatic tumor samples, a distinction was made between diffuse (throughout the whole tumor) or marginal (peripheral staining, on the interface between tumor and stroma) expression of PD-L1 by the tumor cells; scores were given for the presence of PD-L1-positive tumor-infiltrating cells (yes/no), and for immune cells accumulated around tumor fields forming a PD-L1-positive cordon (yes/no). In primary cervical cancer samples, semiquantitative scores were given for PD-L1-positive stromal cells (low numbers/high numbers). In metastatic lymph node samples, scores were obtained for resident lymph node tissue adjacent to metastases (peritumoral) or distant from metastases (paracortical areas) (low numbers/high numbers). Stromal cells and histiocytes present in B-cell follicles were used as an internal control for PD-L1 positivity.

The immunofluorescence was analyzed and imaged using a digital imaging fluorescence microscope (Axiovert-200M; Zeiss, Oberkochen, Germany). Tumor fields were distinguished from normal tissue by the use of DAPI staining.

\section{Statistical Analysis}

The statistical analyses were performed with IBM SPSS (IBM, Armonk, NY, USA) and GraphPad Prism 5 (GraphPad Software, La Jolla, CA, USA). The Pearson's $\chi^{2}$ or Fisher's exact tests were used for the comparison of PD-L1 expression between squamous cell carcinoma and adenocarcinoma, and clinicopathological characteristics. Kaplan-Meier 5-year survival curves were generated and log-rank analyses were performed. Primary tumors and paired metastatic lymph nodes were compared with the McNemar test. $P$-values below 0.05 were considered statistically significant.

\section{Results}

\section{PD-L1 Protein Expression in Primary Cervical Cancer}

Representative examples of different PD-L1 expression patterns in primary cervical tumors (patient cohort I, see Table 1) are depicted in Figure 1 and the results are summarized in Table 3 . We observed PD-L1 positivity in tumor cells, in tumor-infiltrating immune cells, and in stromal immune cells. All tumor-infiltrating and the majority of stromal PD-L1positive immune cells were identified as tumorassociated macrophages, being double positive for CD163 and PD-L1 and/or triple positive for CD163, CD14, and PD-L1 (Figure 2). PD-L1 positivity was observed in $>5 \%$ (used as cutoff) of the tumor cells in $54 \%$ of the squamous cell carcinomas and in $14 \%$ of all adenocarcinomas $(P<0.001)$. In addition, PD-L1-positive tumor-associated macrophages were 

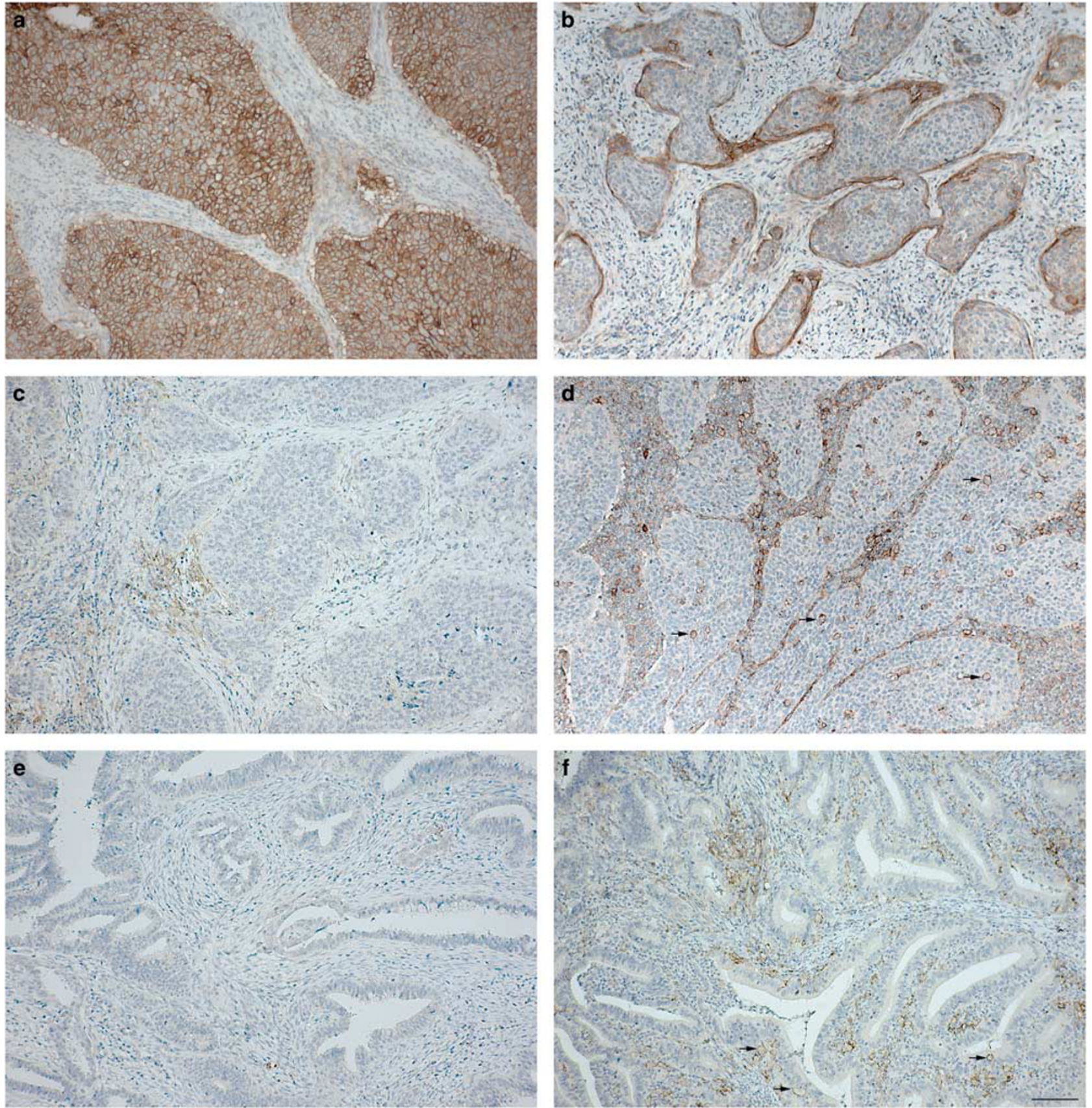

Figure 1 Programmed death-ligand 1 (PD-L1) expression patterns in cervical cancer. Different patterns for PD-L1 expression (in brown) were detected in primary squamous cell carcinoma and adenocarcinoma. (a) Diffuse PD-L1 expression by primary squamous cell carcinoma cells. (b) Marginal PD-L1 expression by primary squamous cell carcinoma cells. (c) PD-L1-negative primary squamous cell carcinoma. (d) Primary squamous cell carcinoma with PD-L1-positive tumor-associated macrophages (examples indicated by black arrows). (e) PD-L1-negative primary adenocarcinoma. (f) PD-L1-negative primary adenocarcinoma with PD-L1-positive tumor-associated macrophages (examples indicated by black arrows). Scale bar is $100 \mu \mathrm{m}$.

present in $53 \%$ of the squamous cell carcinomas and in $12 \%$ of the adenocarcinomas $(P<0.001)$ (Table 3$)$. For stromal PD-L1-positive immune cells and aggregates of PD-L1-positive cells at the tumor stroma interface (termed as PD-L1-positive cordon), no significant differences were found between squamous cell carcinomas and adenocarcinomas.

\section{PD-L1 Expression in Relation to Clinicopathological Characteristics and Survival}

PD-L1 expression was analyzed in relation to clinicopathological characteristics for patient cohort I. Interestingly, we found the majority of PD-L1positive squamous cell carcinoma more often to be 
Table 3 PD-L1 expression in patient cohort I

\begin{tabular}{lrrr}
\hline & SCC & AC & P-value \\
\hline Tumor cells & & & \\
PD-L1+ (>5\%) & $83(54)$ & $7(14)$ & \\
PD-L1 - & $71(46)$ & $42(86)$ & $<\boldsymbol{0 . 0 0 1}$ \\
Diffuse PD-L1 & $71(87)$ & $4(80)$ & \\
Margin PD-L1 & $11(13)^{\mathrm{a}}$ & $1(20)^{\mathrm{a}}$ & 0.533 \\
PD-L1+ TAM & & & \\
Yes & $79(53)$ & $6(12)$ & \\
No & $70(47)$ & $43(88)$ & $<\mathbf{0 . 0 0 1}$ \\
Stromal PD-L1+ cells & & & \\
High numbers & $122(78)$ & $31(65)$ & \\
Low numbers & $34(22)$ & $17(35)$ & 0.057 \\
PD-L1+ cordon & & & \\
Yes & $38(25)$ & $7(15)$ & \\
No & $116(75)$ & $41(85)$ & 0.142 \\
\hline
\end{tabular}

Abbreviations: AC, adenocarcinoma; PD-L1+, PD-L1 positive; PD-L1 - , PD-L1 negative; PD-L1+ TAM, PD-L1-positive TAMs in tumor fields; PD-L1+ cordon, PD-L1-positive immune cells accumulated around tumor fields; SCC, squamous cell carcinoma; TAM, tumor-associated macrophage.

$P$-value was calculated with $\chi^{2}$ test, or in case of $<5$ cases per group with Fisher's exact test.

an some PD-L1-positive tumors ( $n=1$ for SCC and $n=2$ for AC), we found the staining pattern not convincing because of a small tumor field, and excluded those cases for scoring diffuse or marginal expression pattern.

Numbers in bold are statistical significant $(P<0.05)$. Numbers in italic are not statistical significant.

HPV18-positive than HPV16-positive squamous cell carcinomas (83\% HPV18 vs 42\% HPV16; $P<0.001$ ). In squamous cell carcinoma, tumors with over $15 \mathrm{~mm}$ infiltration depth had more often low numbers of PD-L1-positive cells in stroma $(P=0.025)$. In adenocarcinoma, although the group sizes were small, patients with a PD-L1-positive cordon presented with a high FIGO stage ( $>$ IBII) $(P=0.010)$. No further significant correlations were found for PD-L1 positivity and clinicopathological characteristics (tumor size, parametrium invasion, vaginal involvement, and lymph node involvement).

In addition, log-rank tests were performed and Kaplan-Meier plots were generated for disease-free survival and disease-specific survival of the two histological subtypes to assess the correlation with PD-L1 positivity. Squamous cell carcinoma patients with diffuse PD-L1 expression or patients with PD-L1-negative tumors had worse disease-free survival $(P=0.022$ and $P=0.029$, respectively $)$ and disease-specific survival $(P=0.046$ and $P=0.096$, respectively) compared with patients with marginal PD-L1 expression in the primary tumor (Figures 3a and b). In squamous cell carcinoma patients, no significant association was found between PD-L1positive tumor-associated macrophages and survival (Figure 3c), whereas adenocarcinoma patients with PD-L1-positive tumor-associated macrophages had a significantly worse disease-specific survival $(P=0.014)$ compared with adenocarcinoma patients without PD-L1-positive tumor-associated macrophages (Figure 3d).

\section{PD-L1 Expression in Primary Tumor and Paired Metastatic Lymph Node}

Next, we studied PD-L1 expression by immunohistochemistry in patient cohort II with samples available from primary and paired metastatic lymph nodes from patients with squamous cell carcinoma and adenocarcinoma (Table 2). The results for cohort II are summarized in Table 4 and Supplementary Table 1. In the primary tumor, in correspondence to the results obtained in cohort I, squamous cell carcinomas were more often positive for PD-L1 $(P=0.024)$ and had more often PD-L1-positive tumor-associated macrophages $(P=0.012)$. In addition, $25 \%$ of the squamous cell carcinomas had a strong PD-L1-positive cordon, compared with $3 \%$ of the adenocarcinomas $(P=0.012)$ (Table 4).

In the metastatic lymph nodes, PD-L1 positivity was detected in tumor cells, tumor-associated macrophages, immune cells surrounding the metastasis in peritumoral areas, immune cells in resident T-cell areas, and in germinal center histiocytes. Representative examples of PD-L1 expression in metastatic cervical lymph nodes are depicted in Figures 4a and $b$. No significant difference was found in PD-L1 expression patterns between squamous cell carcinoma and adenocarcinoma metastatic lymph nodes (Table 4).

Next, we compared PD-L1 expression between primary tumors and paired metastases. Discordant tumor cell staining of PD-L1 between primary tumor cells and metastatic tumor cells was found for squamous cell carcinomas in 22 of $71(31 \%)$ cases and for adenocarcinomas in 5 of $28(18 \%)$ cases (Supplementary Table 1). Nevertheless, overall in squamous cell carcinoma and adenocarcinoma patients, no significant differences was found between primary tumors and paired metastatic lymph nodes in PD-L1 positivity of tumor cells, diffuse and marginal PD-L1 expression, the presence of PD-L1-positive tumor-associated macrophages, and the presence of a PD-L1-positive cordon (Figures 4c-e and g). In both squamous cell carcinomas and adenocarcinomas, more dense cordons of PD-L1-positive immune cells were found surrounding the metastases compared with the paired primary tumors $(P=0.001$ for squamous cell carcinoma and $P=0.041$ for adenocarcinoma) (Figure $4 \mathrm{f}$ ).

\section{Discussion}

New immunotherapies targeting the PD-1/PD-L1 axis have been reported to give very promising clinical responses in patients with various types of cancer. ${ }^{17,21-24}$ However, until now no data are available on the clinical efficacy of blocking this checkpoint in cervical cancer. PD-L1 positivity has 

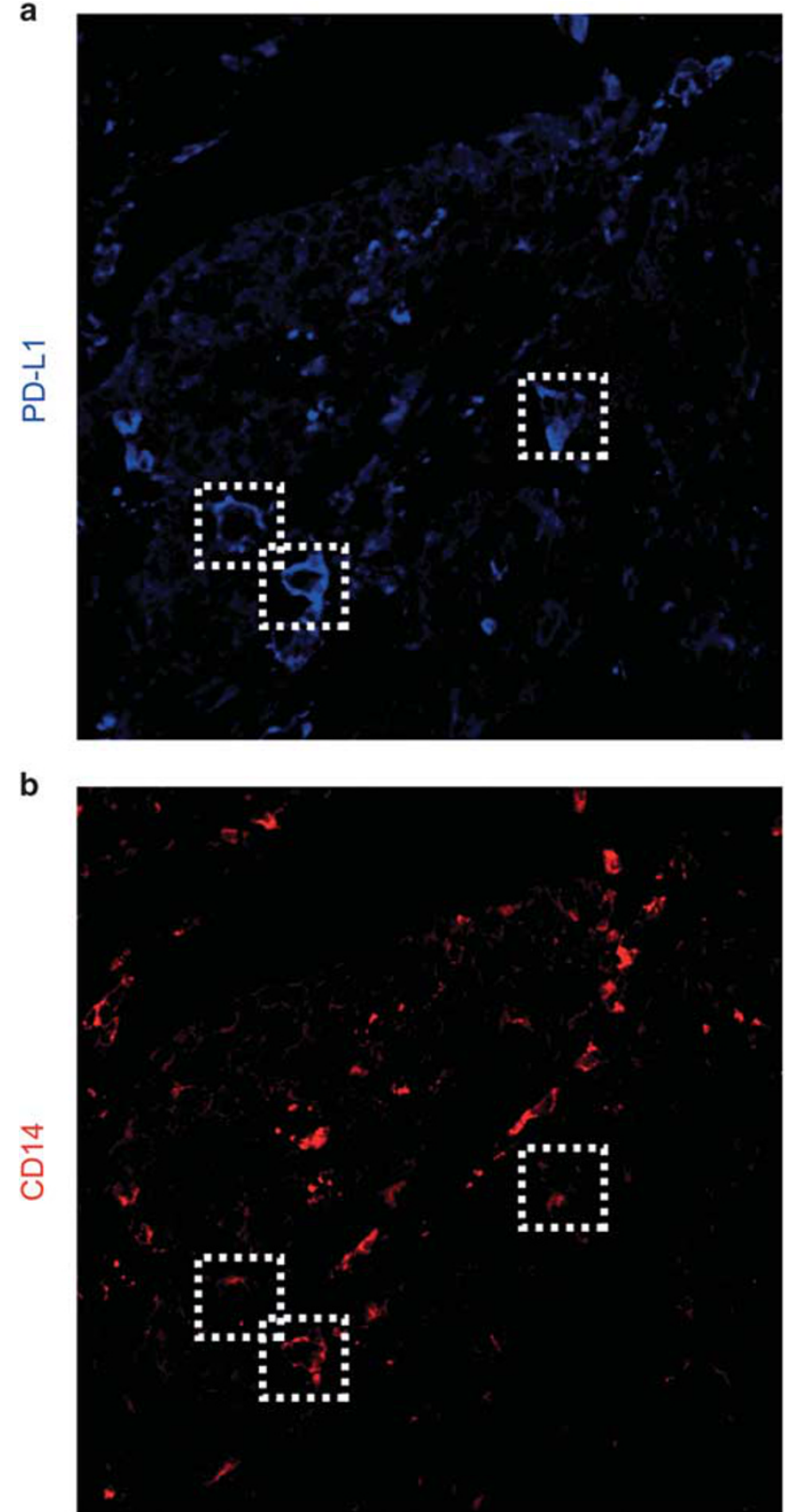

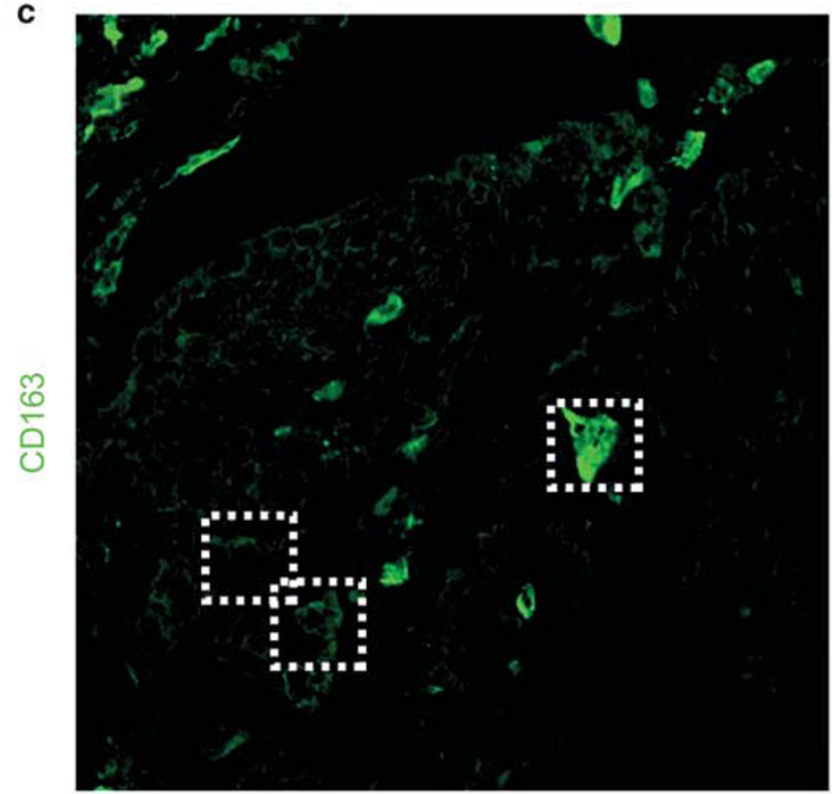

d

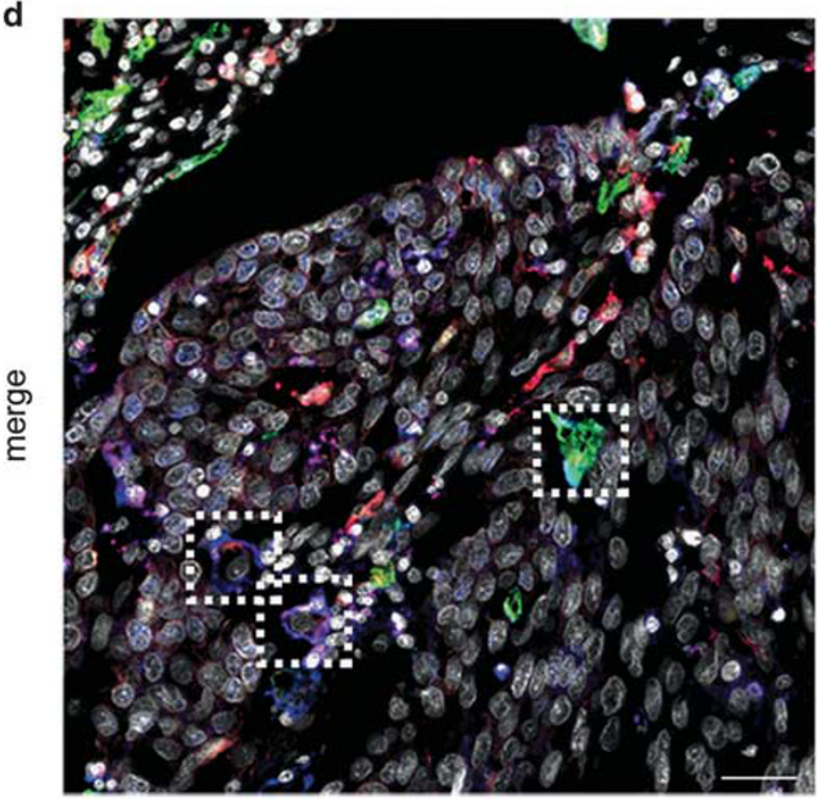

Figure 2 Identification of programmed death-ligand 1 (PD-L1)-positive tumor-associated macrophages. A representative triple immunofluorescence staining shows monochromatic (a) PD-L1, (b) CD14, (c) CD163 images, and (d) colocalized PD-L1 (in blue), CD14 (in red), CD163 (in green), and DAPI (4',6-diamidino-2-phenylindole) (in gray) in cervical cancer patients with PD-L1-positive tumorassociated macrophages in primary tumors. NB: Varying intensity of CD163 staining can be observed. Scale bar is $30 \mu \mathrm{m}$.

been reported previously in cervical intraepithelial neoplasias and cervical carcinomas, ${ }^{25-27}$ and, recently, we have reported on the presence of PD-L1-positive immune cells in tumor-draining lymph nodes, including metastasis-free and metastatic lymph nodes. ${ }^{19,20}$ However, extensive studies on PD-L1 expression in a large patient cohort of primary and paired metastatic cervical cancer samples, in relation to histological subtype and clinicopathological patient characteristics, are lacking.

In the present study, we observed diverse, heterogeneous PD-L1 expression patterns among primary tumors from patients with cervical cancer. Although, there are controversies concerning the use of different PD-L1 antibody clones, several studies have shown that the clones used in the present study are specific and validated for immunohistochemical assays. $^{28,29}$ Apart from the tumor cells, we also observed PD-L1 positivity in immune cells present in the tumor fields and in the stromal compartment. In more than $20 \%$ of the tumors, we observed a PD-L1-positive cordon which was also described in other tumor types. ${ }^{30,31}$ These PD-L1-positive immune cells might have an immunosuppressive effect by inhibiting T-cell function ${ }^{32}$ or might be a sign of immune activation, in conjunction with the 
a
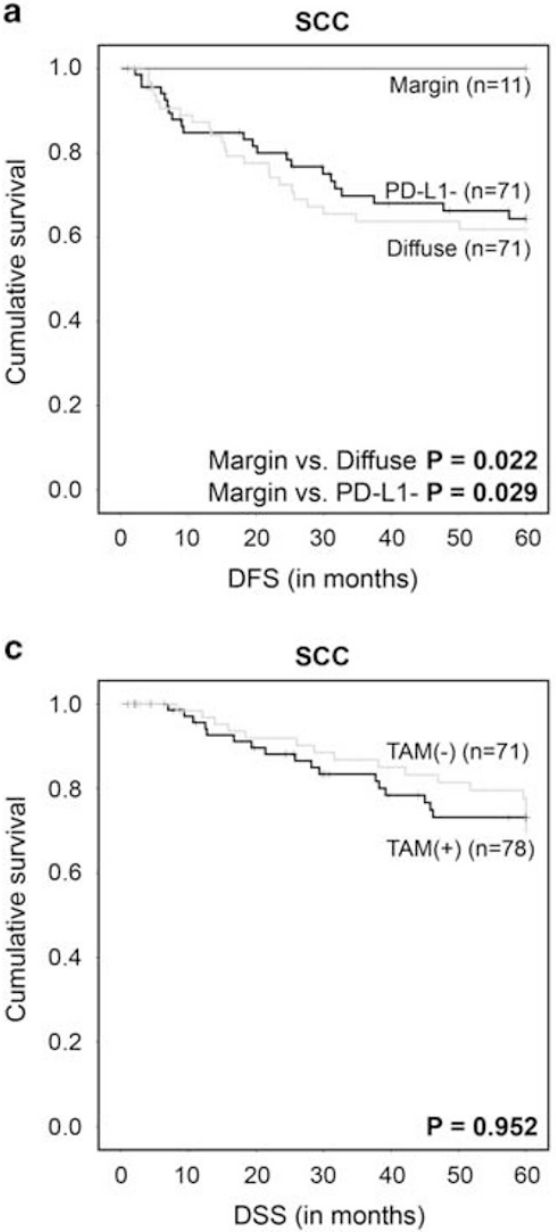

b

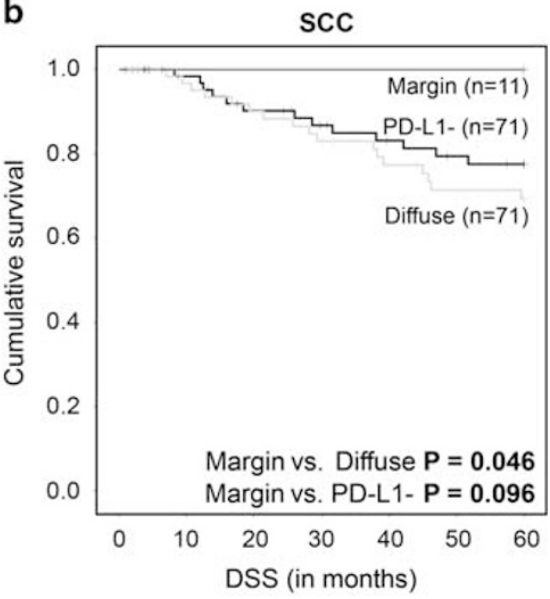

d

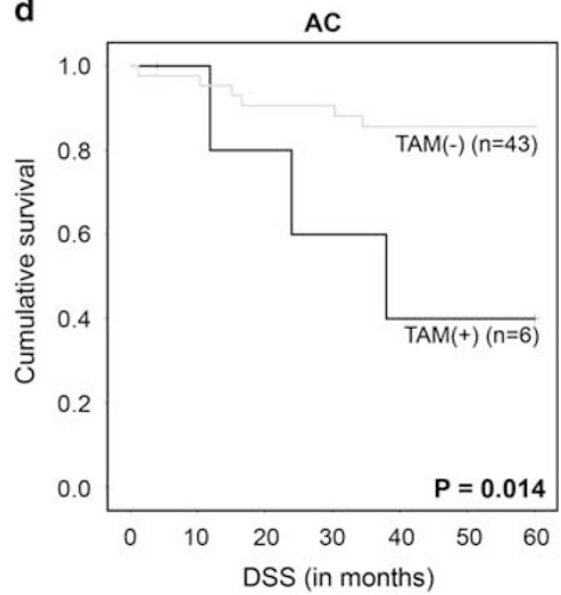

Figure 3 Survival analysis for PD-L1 positivity. Kaplan-Meier 5-year survival curves show disease-free survival (DFS) (a) and diseasespecific survival (DSS) (b) for patients with diffuse PD-L1 expression by tumor cells, for patients with PD-L1-negative (PD-L1 - ) tumors, and for patients with marginal PD-L1 expression in squamous cell carcinoma. Kaplan-Meier 5-year survival curve shows disease-specific survival (DSS) for patients with (c) squamous cell carcinoma and (d) adenocarcinoma with PD-L1-positive tumor-associated macrophages $(\mathrm{TAM}(+))$ and for patients without PD-L1-positive tumor-associated macrophages $(\mathrm{TAM}(-))$. $P$-values were calculated using the log-rank test.

costimulatory markers CD80 and CD86 as expressed on mature dendritic cells. ${ }^{33}$ We identified these PDL1-positive immune cells as $\mathrm{CD}_{163^{+}}$and/or CD14 ${ }^{+}$ tumor-associated macrophages, whereas, remarkably, another study on cervical cancer claimed them to consist mainly of $\mathrm{CD}^{+} \mathrm{T}$ cells. ${ }^{27}$ The presence of PD-L1 ${ }^{+} \mathrm{T}$ cells was also reported by other studies; however, in these studies compelling evidence in the form of double stainings was lacking, ${ }^{17,34}$ and, therefore, it is more likely that PD-L1-positive tumorinfiltrating cells are from myeloid origin with an M2 macrophage-like phenotype as observed by us, which is in accordance with multiple other studies. ${ }^{31,35,36}$ Similar M2-like cells, conditioned by tumor-derived soluble factors, have been shown to be poor CD8 ${ }^{+}$ T-cell primers, potent inducers of $\mathrm{FoxP}^{+}$regulatory $\mathrm{T}$ cells and proangiogenic- and protumor-invasive factor producers facilitating tumor progression. ${ }^{37,38}$ Although different myeloid cell sub-populations and a low CTL/regulatory T-cell ratio have been found to correlate to survival in the cervical tumor micro- environment, ${ }^{39,40}$ the precise role of PD-L1-positive tumor-associated macrophages is yet to be fully elucidated. Nevertheless, in vitro observations by Heusinkveld et al., ${ }^{41}$ suggest that cervical cancerderived IL-6 and prostaglandin-E2 convert monocytes to T-cell-tolerizing macrophages with low levels of costimulatory molecules and IL-12p70, and high levels of IL-10 and PD-L1 expression consistent with a poor ability to prime naïve T cells. In accordance, we have previously shown that high IL-6 in the tumor microenvironment of cervical cancer is associated with poor patient survival. ${ }^{42,43}$

This is the first study to report on the difference in PD-L1 expression between squamous cell carcinoma and adenocarcinoma. Two previous publications on PD-L1 expression in cervical cancer did not include adenocarcinoma patients in the cohorts analyzed. ${ }^{25,27}$ Strikingly, we observed prognostic differences for PD-L1 expression patterns between squamous cell carcinoma and adenocarcinoma patients; we found significantly more PD-L1 
Table 4 PD-L1 expression in patient cohort II

\begin{tabular}{|c|c|c|c|c|c|c|}
\hline & \multicolumn{3}{|c|}{ Primary tumor } & \multicolumn{3}{|c|}{ Metastatic tumor } \\
\hline & $S C C$ & $A C$ & $\mathrm{P}$-value & $S C C$ & $A C$ & $\mathrm{P}$-value \\
\hline \multicolumn{7}{|l|}{ Tumor cells } \\
\hline PD-L1+ $(>5 \%)$ & $33(39)$ & 5 (17) & & $21(26)$ & $3(10)$ & \\
\hline PD-L1 - & $51(61)$ & $25(83)$ & 0.024 & $60(74)$ & $26(90)$ & 0.116 \\
\hline Diffuse PD-L1 & $27(82)$ & $4(100)^{\mathrm{a}}$ & & $12(63)$ & $2(67)$ & \\
\hline Margin PD-L1 & $6(18)$ & $0(0)$ & 1 & 7 (37) & $1(33)$ & 1 \\
\hline \multicolumn{7}{|l|}{$P D-L 1+T A M$} \\
\hline Yes & $24(29)$ & $2(7)$ & & $21(26)$ & $3(10)$ & \\
\hline No & $59(71)$ & $28(93)$ & 0.012 & $60(74)$ & $26(90)$ & 0.116 \\
\hline \multicolumn{7}{|c|}{ Peritumoral PD-L1+ cells } \\
\hline High numbers & $40(49)$ & $11(37)$ & & $62(77)$ & $22(76)$ & \\
\hline Low numbers & $42(51)$ & $19(63)$ & 0.254 & $19(23)$ & $7(24)$ & 1 \\
\hline \multicolumn{7}{|l|}{ PD-L1+ cordon } \\
\hline Yes & $20(25)$ & $1(3)$ & & $20(25)$ & $4(14)$ & \\
\hline No & $61(75)$ & $28(97)$ & 0.012 & $61(75)$ & $24(86)$ & 0.301 \\
\hline
\end{tabular}

Abbreviations: AC, adenocarcinoma; PD-L1+, PD-L1 positive; PD-L1 PD-L1 negative; PD-L1+ TAM, PD-L1-positive TAMs in tumor fields; peritumoral immune cells, PD-L1-positive immune cells in the vicinity of metastatic tumor fields; PD-L1+ cordon, PD-L1-positive immune cells accumulated around tumor fields; SCC, squamous cell carcinoma; TAM, tumor-associated macrophage.

$P$-value was calculated with $\chi^{2}$ test, or in case of $<5$ cases per group with Fisher's exact test.

${ }^{\text {In }}$ one AC PD-L1+ tumor, we found the staining pattern not convincing because of a small tumor field, and excluded this case for scoring diffuse or marginal expression pattern.

Numbers in bold are statistical significant $(P<0.05)$. Numbers in italic are not statistical significant.

expression by tumor cells (cutoff $>5 \%$ ) and higher rates of PD-L1-positive tumor-associated macrophages in squamous cell carcinomas as compared with adenocarcinomas. Similarly, differential findings for PD-L1 expression in the two histological subtypes were reported in lung cancer patients. ${ }^{35,44}$

Earlier studies have reported conflicting data on correlations between PD-L1 expression in different solid tumor types with both improved ${ }^{30,45,46}$ and poor prognosis. ${ }^{4-51}$ However, recent meta-analyses have shown a predominant correlation with poor survival. ${ }^{52-54}$ We were not able to detect an association between PD-L1 expression per se and survival, which is in accordance with an earlier study in patients with cervical cancer, analyzing the whole cohort through the use of tissue microarrays. ${ }^{25} \mathrm{Of}$ note, we did find an unambiguous survival benefit for squamous cell carcinoma patients with marginal PD-L1 tumor expression (at the tumor-stromal interface) as compared with patients with diffusely positive PD-L1 tumors. In head and neck cancer, diffuse PD-L1 expression was detected in only 1/14 tumors, whereas marginal PD-L1 expression was detected in 13/14 tumors, but no survival analysis was performed. ${ }^{31}$ Marginal PD-L1 expression might be induced by extrinsic factors, such as IFN $\gamma$, TNF $\alpha$, and IL-1 $\beta$ locally produced by juxtaposed $\mathrm{T}$ lymphocytes, whereas diffuse PD-L1 expression is more likely to result from constitutive expression because of underlying tumor-intrinsic molecular mechanisms such as PTEN loss and aberrant JAK/ STAT signaling. ${ }^{24,30,55}$ Importantly, conjunction with infiltrating effector $\mathrm{T}$ cells and the release of type-1 effector cytokines might explain the observed association between marginal expression of PD-L1 and a more favorable prognosis (see Figures 3a and b). Recently, we reported on a survival benefit for cervical cancer patients with high numbers of Tbet-positive $\mathrm{T}$ cells, indicative of high $\mathrm{IFN} \gamma$ production. ${ }^{56}$

In adenocarcinoma, we observed a survival benefit for patients with tumor lacking PD-L1-positive tumorassociated macrophages. These findings point to a difference in immunological microenvironments and tumor escape mechanisms between cervical adenocarcinoma and squamous cell carcinoma in line with previous reports on histology-specific oncogenic mutations, ${ }^{3,4}$ and immunological profiles. ${ }^{5-7}$

Our findings suggest that targeting the PD-1/PD-L1 pathway might be a promising immunotherapy approach in patients with cervical cancer, as PD-L1 is expressed in 54\% of the squamous cell carcinomas. In addition, recent studies have shown that even patients with PD-L1-negative primary tumors, including lung cancer, gastric cancer, colorectal cancer, renal cell cancer, and bladder cancer and melanoma, respond to anti-PD-L1 treatment. ${ }^{18,24,57}$ This might be due to the observed heterogeneous and discordant PD-L1 tumor cell staining between primary tumor cells and metastatic tumor cells with, in some cases, PD-L1-positive metastases originating from PD-L1-negative primary tumors (see Supplementary Table 1), which was also observed in clear-cell renal cell carcinoma. ${ }^{58,59}$ Adenocarcinoma patients with PD-L1-positive tumor-associated macrophages had a poor survival; however, antiPD-1 or anti-PD-L1 therapy might be successful, as in bladder cancer patients with PD-L1-positive tumorinfiltrating immune cells objective responses were obtained after anti-PD-L1 therapy. ${ }^{18}$ Of note, patient stratification on the basis of PD-L1-positive tumorassociated macrophages is very important in this regard, as patients with adenocarcinoma infiltrated by PD-L1-positive tumor-associated macrophages represented a relatively small minority (see Table 4).

In conclusion, this study showed that PD-L1 was more frequently expressed by squamous cell carcinoma than by adenocarcinoma. Diffuse PD-L1 expression in squamous cell carcinoma patients was correlated with poor disease-free survival and disease-specific survival compared with marginal PD-L1 expression, which was associated with a remarkably favorable prognosis. In adenocarcinoma patients, the presence of PD-L1-positive tumorassociated macrophages was associated with a poor disease-specific survival as compared with patients without PD-L1-positive tumor-associated macrophages. Our data thus suggest that targeting the PD-1/PD-L1 pathway may be therapeutically efficacious and should be considered in the treatment of cervical cancer patients. 


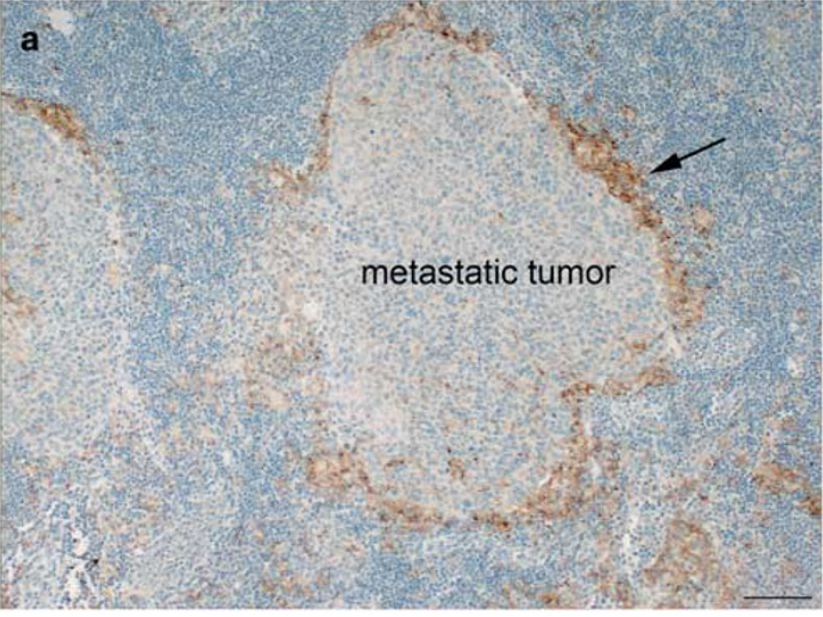

c

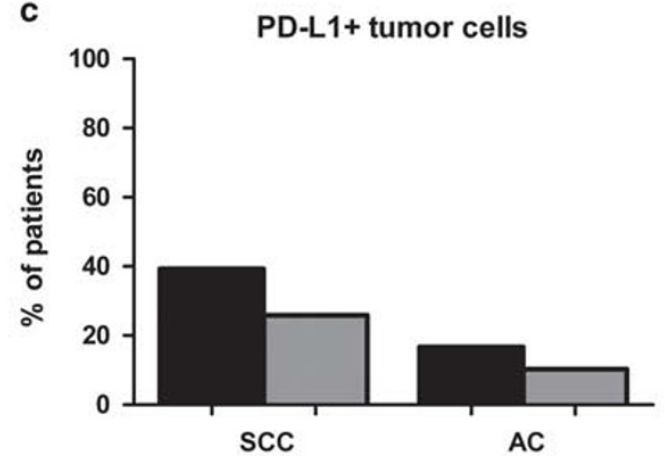

e

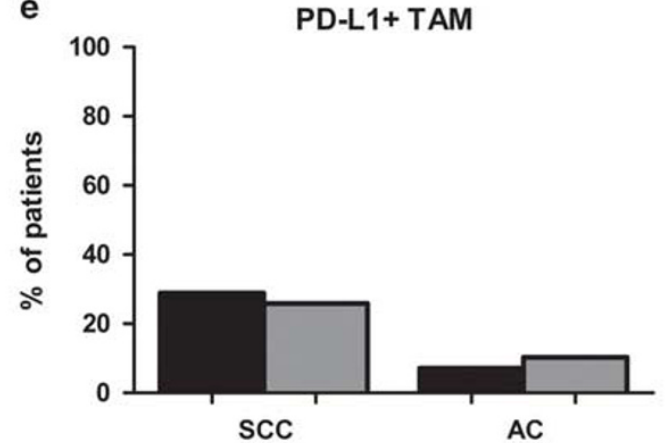

g

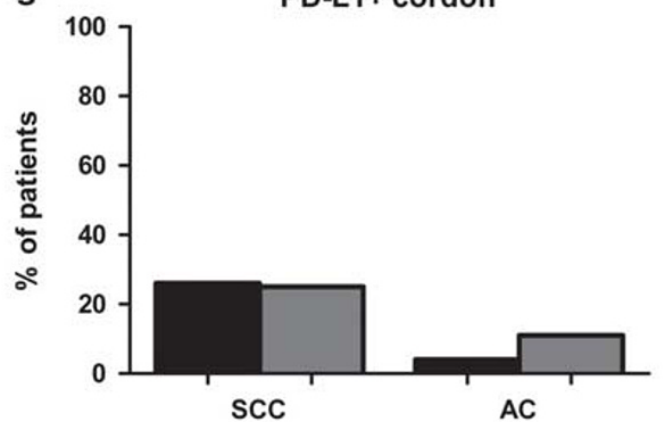

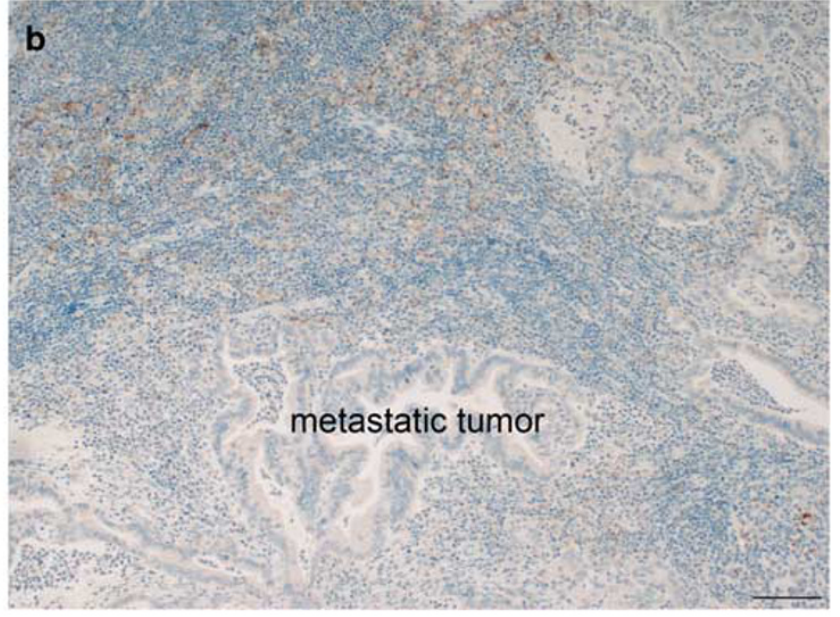

d

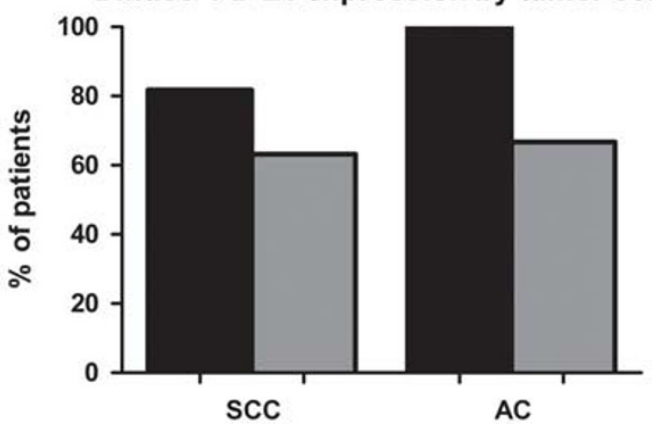

f

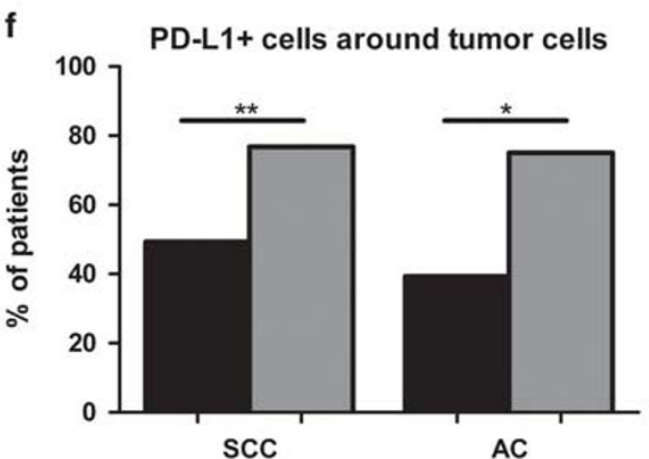

Figure 4 PD-L1 expression in metastatic lymph nodes. PD-L1 (in brown) expression in (a) metastatic squamous cell carcinoma with a PDL1-positive cordon indicated by the black arrow and high numbers of PD-L1-positive peritumoral immune cells, and (b) metastatic adenocarcinoma lymph node samples. PD-L1 positivity in primary squamous cell carcinoma (SCC) and adenocarcinoma (AC) and metastatic lymph nodes (c) with PD-L1-positive tumor cells, (d) with diffuse PD-L1 expression, (e) with the presence of PD-L1-positive tumor-associated macrophages (TAM), (f) with the presence of peritumoral PD-L1-positive immune cells, and (g) with the presence of a PD-L1-positive cordon. ${ }^{*} P=0.001$ and ${ }^{*} P=0.041$ calculated with McNemar test. Scale bar is $50 \mu \mathrm{m}$. 


\section{Acknowledgments}

We thank Wim Vos for technical assistance, JeroenWierda for assisting with the PD-L1 scoring, Dr Wessel van Wieringen for statistical advice, Dr René Musters for the use of the fluorescence microscopy facility, and Dr. Sanne Samuels and Dr. Debbie Ferns for patient inclusion. This work was supported by a research grant from the Dutch Cancer Society (KWF VU 2013-6015).

\section{Disclosure/conflict of interest}

The authors declare no conflict of interest.

\section{References}

1 de Freitas AC, Gurgel AP, Chagas BS et al. Susceptibility to cervical cancer: an overview. Gynecol Oncol 2012;126:304-311.

2 Ferlay J, Soerjomataram I, Ervik $M$ et al. GLOBOCAN 2012 v.1.0, Cancer Incidence and Mortality Worldwide: IARC CancerBase No. 11 [Internet]. International Agency for Research on Cancer: Lyon, France, 2013.

3 Wright AA, Howitt BE, Myers AP et al. Oncogenic mutations in cervical cancer: genomic differences between adenocarcinomas and squamous cell carcinomas of the cervix. Cancer 2013;119:3776-3783.

4 Spaans VM, Trietsch MD, Peters AA et al. Precise classification of cervical carcinomas combined with somatic mutation profiling contributes to predicting disease outcome. PLoS One 2015;10:e0133670.

5 Spaans VM, Peters AA, Fleuren GJ et al. HLA-E expression in cervical adenocarcinomas: association with improved long-term survival. J Transl Med 2012;10:184.

6 Samuels S, Ferns DM, Meijer D et al. High levels of soluble MICA are significantly related to increased disease-free and disease-specific survival in patients with cervical adenocarcinoma. Tissue Antigens 2015;85:476-483.

7 Punt S, van Vliet ME, Spaans VM et al. FoxP3 and IL-17 cells are correlated with improved prognosis in cervical adenocarcinoma. Cancer Immunol Immunother 2015; 64:745-753.

8 Waggoner SE. Cervical cancer. Lancet 2003;361: 2217-2225.

9 Rose PG. Chemoradiotherapy for cervical cancer. Eur J Cancer 2002;38:270-278.

10 Suprasert P, Srisomboon J, Kasamatsu T. Radical hysterectomy for stage IIB cervical cancer: a review. Int J Gynecol Cancer 2005;15:995-1001.

11 Shingleton HM, Bell MC, Fremgen A et al. Is there really a difference in survival of women with squamous cell carcinoma, adenocarcinoma, and adenosquamous cell carcinoma of the cervix?. Cancer 1995;76:1948-1955.

12 Smith HO, Tiffany MF, Qualls CR et al. The rising incidence of adenocarcinoma relative to squamous cell carcinoma of the uterine cervix in the United Statesa 24-year population-based study. Gynecol Oncol 2000;78:97-105.

13 Yamauchi M, Fukuda T, Wada T et al. Comparison of outcomes between squamous cell carcinoma and adenocarcinoma in patients with surgically treated stage I-II cervical cancer. Mol Clin Oncol 2014;2:518-524.
14 Biewenga P, van d V, Mol BW et al. Prognostic model for survival in patients with early stage cervical cancer. Cancer 2011;117:768-776.

15 Sharma P, Allison JP. The future of immune checkpoint therapy. Science 2015;348:56-61.

16 Pennock GK, Chow LQ. The evolving role of immune checkpoint inhibitors in cancer treatment. Oncologist 2015;20:812-822.

17 Herbst RS, Soria JC, Kowanetz M et al. Predictive correlates of response to the anti-PD-L1 antibody MPDL3280A in cancer patients. Nature 2014;515:563-567.

18 Powles T, Eder JP, Fine GD et al. MPDL3280A (anti-PDL1) treatment leads to clinical activity in metastatic bladder cancer. Nature 2014;515:558-562.

19 Heeren AM, Koster BD, Samuels S et al. High and interrelated rates of PD-L1+CD14+ antigen-presenting cells and regulatory $\mathrm{T}$ cells mark the microenvironment of metastatic lymph nodes from patients with cervical cancer. Cancer Immunol Res 2015;3:48-58.

20 Heeren AM, de BE, Bleeker MC et al. Nodal metastasis in cervical cancer occurs in clearly delineated fields of immune suppression in the pelvic lymph catchment area. Oncotarget 2015;6:32484-32493.

21 Topalian SL, Hodi FS, Brahmer JR et al. Safety, activity, and immune correlates of anti-PD-1 antibody in cancer. N Engl J Med 2012;366:2443-2454.

22 Carosella ED, Ploussard G, LeMaoult J et al. A systematic review of immunotherapy in urologic cancer: evolving roles for targeting of CTLA-4, PD-1/PD-L1, and HLA-G. Eur Urol 2015;68:267-279.

23 Soria JC, Marabelle A, Brahmer JR et al. Immune checkpoint modulation for non-small cell lung cancer. Clin Cancer Res 2015;21:2256-2262.

24 Patel SP, Kurzrock R. PD-L1 expression as a predictive biomarker in cancer immunotherapy. Mol Cancer Ther 2015;14:847-856.

25 Karim R, Jordanova ES, Piersma SJ et al. Tumorexpressed B7-H1 and B7-DC in relation to PD-1+ T-cell infiltration and survival of patients with cervical carcinoma. Clin Cancer Res 2009;15:6341-6347.

26 Yang W, Song Y, Lu YL et al. Increased expression of programmed death (PD)-1 and its ligand PD-L1 correlates with impaired cell-mediated immunity in highrisk human papillomavirus-related cervical intraepithelial neoplasia. Immunology 2013;139:513-522.

27 Mezache L, Paniccia B, Nyinawabera A et al. Enhanced expression of PD L1 in cervical intraepithelial neoplasia and cervical cancers. Mod Pathol 2015;28: 1594-1602.

28 Teixido C, Karachaliou N, Gonzalez-Cao $\mathrm{M}$ et al. Assays for predicting and monitoring responses to lung cancer immunotherapy. Cancer Biol Med 2015;12: 87-95.

29 Schats K, van Vré S, de Schepper S et al. PD-L1 IHC validation and comparison of E1L3N \& SP142 mAbs in melanoma [poster]. Ann Oncol 2015;26.

30 Taube JM, Anders RA, Young GD et al. Colocalization of inflammatory response with B7-h1 expression in human melanocytic lesions supports an adaptive resistance mechanism of immune escape. Sci Transl Med 2012;4:127ra37.

31 Lyford-Pike S, Peng S, Young GD et al. Evidence for a role of the PD-1:PD-L1 pathway in immune resisstance of HPV-associated head and neck squamous cell carcinoma. Cancer Res 2013;73:1733-1741.

32 Iwai Y, Ishida M, Tanaka Y et al. Involvement of PD-L1 on tumor cells in the escape from host immune system 
and tumor immunotherapy by PD-L1 blockade. Proc Natl Acad Sci USA 2002;99:12293-12297.

33 van de Ven $\mathrm{R}$, van den Hout MF, Lindenberg JJ et al. Characterization of four conventional dendritic cell subsets in human skin-draining lymph nodes in relation to T-cell activation. Blood 2011;118: 2502-2510.

34 Ghebeh H, Mohammed S, Al-Omair A et al. The B7-H1 (PD-L1) T lymphocyte-inhibitory molecule is expressed in breast cancer patients with infiltrating ductal carcinoma: correlation with important high-risk prognostic factors. Neoplasia 2006;8:190-198.

$35 \mathrm{Mu} \mathrm{CY}$, Huang JA, Chen Y et al. High expression of PDL1 in lung cancer may contribute to poor prognosis and tumor cells immune escape through suppressing tumor infiltrating dendritic cells maturation. Med Oncol 2011;28:682-688.

36 Schultheis AM, Scheel AH, Ozretic L et al. PD-L1 expression in small cell neuroendocrine carcinomas. Eur J Cancer 2015;51:421-426.

37 Lindenberg JJ, van d V, Lougheed SM et al. Functional characterization of a STAT3-dependent dendritic cellderived CD14 cell population arising upon IL-10driven maturation. Oncoimmunology 2013;2:e23837.

38 van de Ven R, Lindenberg JJ, Oosterhoff $\mathrm{D}$ et al. Dendritic cell plasticity in tumor-conditioned skin: CD14(+) cells at the cross-roads of immune activation and suppression. Front Immunol 2013;4:403.

39 Jordanova ES, Gorter A, Ayachi O et al. Human leukocyte antigen class I, MHC class I chain-related molecule A, and CD8+/regulatory T-cell ratio: which variable determines survival of cervical cancer patients?. Clin Cancer Res 2008;14:2028-2035.

40 de Vos van Steenwijk PJ, Ramwadhdoebe TH, Goedemans R et al. Tumor-infiltrating CD14-positive myeloid cells and CD8-positive T-cells prolong survival in patients with cervical carcinoma. Int J Cancer 2013;133: 2884-2894.

41 Heusinkveld M, de Vos van Steenwijk PJ, Goedemans R et al. M2 macrophages induced by prostaglandin E2 and IL-6 from cervical carcinoma are switched to activated M1 macrophages by CD4+ Th1 cells. J Immunol 2011;187:1157-1165.

42 Punt S, Houwing-Duistermaat JJ, Schulkens IA et al. Correlations between immune response and vascularization qRT-PCR gene expression clusters in squamous cervical cancer. Mol Cancer 2015;14:71.

43 Zijlmans HJ, Punt S, Fleuren GJ et al. Role of IL-12p40 in cervical carcinoma. Br J Cancer 2012;107:1956-1962.

44 Konishi J, Yamazaki K, Azuma $\mathrm{M}$ et al. B7-H1 expression on non-small cell lung cancer cells and its relationship with tumor-infiltrating lymphocytes and their PD-1 expression. Clin Cancer Res 2004;10: $5094-5100$

45 Velcheti V, Schalper KA, Carvajal DE et al. Programmed death ligand-1 expression in non-small cell lung cancer. Lab Invest 2014;94:107-116.

46 Cooper WA, Tran T, Vilain RE et al. PD-L1 expression is a favorable prognostic factor in early stage non-small cell carcinoma. Lung Cancer 2015;89:181-188.

47 Thompson RH, Kuntz SM, Leibovich BC et al. Tumor B7-H1 is associated with poor prognosis in renal cell carcinoma patients with long-term follow-up. Cancer Res 2006;66:3381-3385.

$48 \mathrm{Wu} \mathrm{C}$, Zhu Y, Jiang J et al. Immunohistochemical localization of programmed death-1 ligand-1 (PD-L1) in gastric carcinoma and its clinical significance. Acta Histochem 2006;108:19-24.

49 Huang Y, Zhang SD, McCrudden C et al. The prognostic significance of PD-L1 in bladder cancer. Oncol Rep 2015;33:3075-3084.

50 Chevolet I, Speeckaert R, Schreuer M et al. Characterization of the immune network of IDO, tryptophan metabolism, PD-L1, and in circulating immune cells in melanoma. Oncoimmunology 2015;4:e982382.

51 Kiyasu J, Miyoshi H, Hirata A et al. Expression of programmed cell death ligand 1 is associated with poor overall survival in patients with diffuse large B-cell lymphoma. Blood 2015;126:2193-2201.

52 Zhang Y, Kang S, Shen J et al. Prognostic significance of programmed cell death 1 (PD-1) or PD-1 ligand 1 (PD-L1) expression in epithelial-originated cancer: a metaanalysis. Medicine (Baltimore, MD) 2015;94:e515.

$53 \mathrm{Wu} \mathrm{P}, \mathrm{Wu} \mathrm{D}, \mathrm{Li}$ L et al. PD-L1 and survival in solid tumors: a meta-analysis. PLoS One 2015;10:e0131403.

54 Jin Y, Zhao J, Shi X et al. Prognostic value of programed death ligand 1 in patients with solid tumors: a metaanalysis. J Cancer Res Ther 2015;11:C38-C43.

55 Ritprajak P, Azuma M. Intrinsic and extrinsic control of expression of the immunoregulatory molecule PD-L1 in epithelial cells and squamous cell carcinoma. Oral Oncol 2015;51:221-228.

56 Gorter A, Prins F, van DM et al. The tumor area occupied by Tbet+ cells in deeply invading cervical cancer predicts clinical outcome. J Transl Med 2015;13: 295.

57 Carbognin L, Pilotto S, Milella M et al. Differential activity of Nivolumab, Pembrolizumab and MPDL3280A according to the tumor expression of programmed death-ligand-1 (PD-L1): sensitivity analysis of trials in melanoma, lung and genitourinary cancers. PLoS One 2015;10:e0130142.

58 Jilaveanu LB, Shuch B, Zito CR et al. PD-L1 expression in clear cell renal cell carcinoma: an analysis of nephrectomy and sites of metastases. J Cancer 2014;5: 166-172.

59 Callea M, Albiges L, Gupta $\mathrm{M}$ et al. Differential expression of PD-L1 between primary and metastatic sites in clear-cell renal cell carcinoma. Cancer Immunol Res 2015;3:1158-1164.

(c) () $\Theta$ This work is licensed under a Creative Commons Attribution-NonCommercialNoDerivs 4.0 International License. The images or other third party material in this article are included in the article's Creative Commons license, unless indicated otherwise in the credit line; if the material is not included under the Creative Commons license, users will need to obtain permission from the license holder to reproduce the material. To view a copy of this license, visit http://creativecommons.org/licenses/ by-nc-nd/4.0/

Supplementary Information accompanies the paper on Modern Pathology website (http://www.nature.com/ modpathol) 